\title{
Centre océanologique du Pacifique
}

In: Journal de la Société des océanistes. º48, Tome 31, 1975. pp. 323-329.

Citer ce document / Cite this document :

de Chazeaux Jean. Centre océanologique du Pacifique. In: Journal de la Société des océanistes. N48, Tome 31, 1975. pp. 323-329.

doi : $10.3406 /$ jso. 1975.2716

http://www.persee.fr/web/revues/home/prescript/article/jso_0300-953X_1975_num_31_48_2716 


\section{Centre océanologique du Pacifique}

Le COP, Centre océanologique du Pacifique est installé depuis 1972 à Vairao, Tahiti, Polynésie Française. Tél. 4 à Vairao; 2.90 .52 à Papeete.

Avec le Centre Océanologique de Bretagne et la Base Océanologique de Méditerranée, le COP est une des trois réalisations françaises du Centre National pour l'Exploitation des océans, CNEXO, établissement public et commercial, créé par la loi du 3 janvier 1967, doté de l'autonomie financière sous le contrôle du Ministre de l'Industrie et de la Recherche. Il est " chargé de développer la connaissance des océans, et les études et recherches tendant à l'exploitation des ressources contenues à leur surface, dans leur masse, leur sol et leur sous-sol n.

Un protocole de cinq ans, renouvelable, lie le CNEXO et le Territoire de la Polynésie Française : il associe les deux parties dans le développement de l'Aquaculture et de la pêche et fixe les règles de la coopération. Des contrats cadre sont ensuite intervenus pour les opérations conduites en commun " aquaculture de chevrettes ", " développement de la pêche à l'appât vivant " et bientôt " nutrition des animaux marins et terrestres ".

Le COP a recherché aussi d'autres collaborations. Et c'est ainsi qu'ont été amenés à participer à l'étude du lagon de Vairao, le Service Mixte de Contrôle Biologique du CEA, l'Institut de Recherches Médicales de Polynésie, l'ORSTOM (Centre de Nouméa), le Muséum National d'Histoire Naturelle et la Faculté de Nice.

À Takapoto, le Service de la Pêche, l'ORSTOM et le COP conjuguent leurs efforts pour dresser le bilan des ressources possibles du lagon, cible également retenue par le projet MAB (Man and Biosphere) qu'anime localement le Pr. Salvat.

Personnel. - Le COP est dirigé par M. Jean de Chazeaux. Les opérations d'aquaculture et de pêche sont effectuées sous la responsabilité de M. Alain Michel. En 1975 les tâches étaient ainsi réparties : Mise au point des techniques d'élevage et unités pilotes : MM. J.-M. Griessinger, Landret, V. Vanaa, F. Fallourd, A. Maillon. Nutrition : MM. Cuzon, Coatanea, Peignon, de la Pomelie. Algues unicellulaires et zooplancton : MM. J.-L. Martin, Millous, Lucain. Pathologie : M. Le Bitoux. Pêche : MM. Bard et Bessineton. Mesures : M. Calvas. Technologie : M. Virmaux. 
Installations. - La rade de Vairao a été choisie comme offrant un plan d'eau bien protégé, d'une température favorable $\left(26-28^{\circ}\right)$ exempt de toute pollution et convenant particulièrement à l'élevage marin. Les principales installations comprennent, outre les bureaux de l'administration et des chercheurs, un grand hall d'expérimentation destiné à aborder tous les problèmes à l'échelle réduite; des bassins de prégrossissement, trois bassins de grossissement $\left(2600 \mathrm{~m}^{2}\right.$, puis 1 hectare), trois laboratoires, une station de pompage, etc.

Financement. - Le Centre a joui en 1974 d'un budget de plus de 7 millions de Francs français.

Publications. - Le COP a très peu diffusé d'informations concernant les travaux d'aquaculture et de pêche de 1973 à 1975 : la raison en est la difficulté à tirer des synthèses de travaux sur une période aussi courte. - Les seuls documents sont à usage interne essentiellement.

Dans l'avenir, ils pourront être obtenus au BNDO (Bureau National des Données Océaniques) à Brest (BP 337 - CEDEX 29273 , Brest) ainsi qu'au Service de l'Information du CNEXO, 39, avenue d'Iéna, 75016 Paris).

\section{ACTIVITÉS}

Le COP est né le $1^{\text {er }}$ octobre 1972. Les constructions seront achevées en 1978, compte tenu de la nécessité de profiter des enseignements acquis au cours des expériences.

Les opérations que le COP a pris en charge de conduire concernent essentiellement le développement de l'aquaculture tropicale et de la pêche au thon. Il apporte son soutien logistique aux campagnes à la mer et particulièrement aux campagnes d'exploration entreprises en vue de rechercher des gisements de nodules polymétalliques. Enfin, il s'efforce de promouvoir l'utilisation des énergies naturelles que peuvent procurer le soleil, le vent et les courants.

C'est en aquaculture que les premiers résultats significatifs ont été obtenus. Il ne sera donc question ici que de dresser le bilan de deux années et dimie d'efforts.

L'aquaculture, en règle générale, comporte plusieurs étapes : la réussite d'un cycle biotechniquement complet comportant la maturation et la reproduction en enceintes contrôlées, puis l'élevage larvaire, le grossissement de sujets commercialisables et la sélection des reproducteurs qui participeront au cycle suivant. La répétitivité doit être acquise avec des productions au niveau souhaité avant de pouvoir aborder tout développement à l'échelle industrielle. En outre, l'aquaculteur doit être en mesure de prévenir les maladies, plutôt que de songer à les guérir. Enfin, il est nécessaire de maîtriser l'eau dans ses qualités biologiques, d'effectuer des mesures de tout genre, produire la nourriture nécessaire à l'harmonieuse croissance de chaque espèce, à chaque stade. 


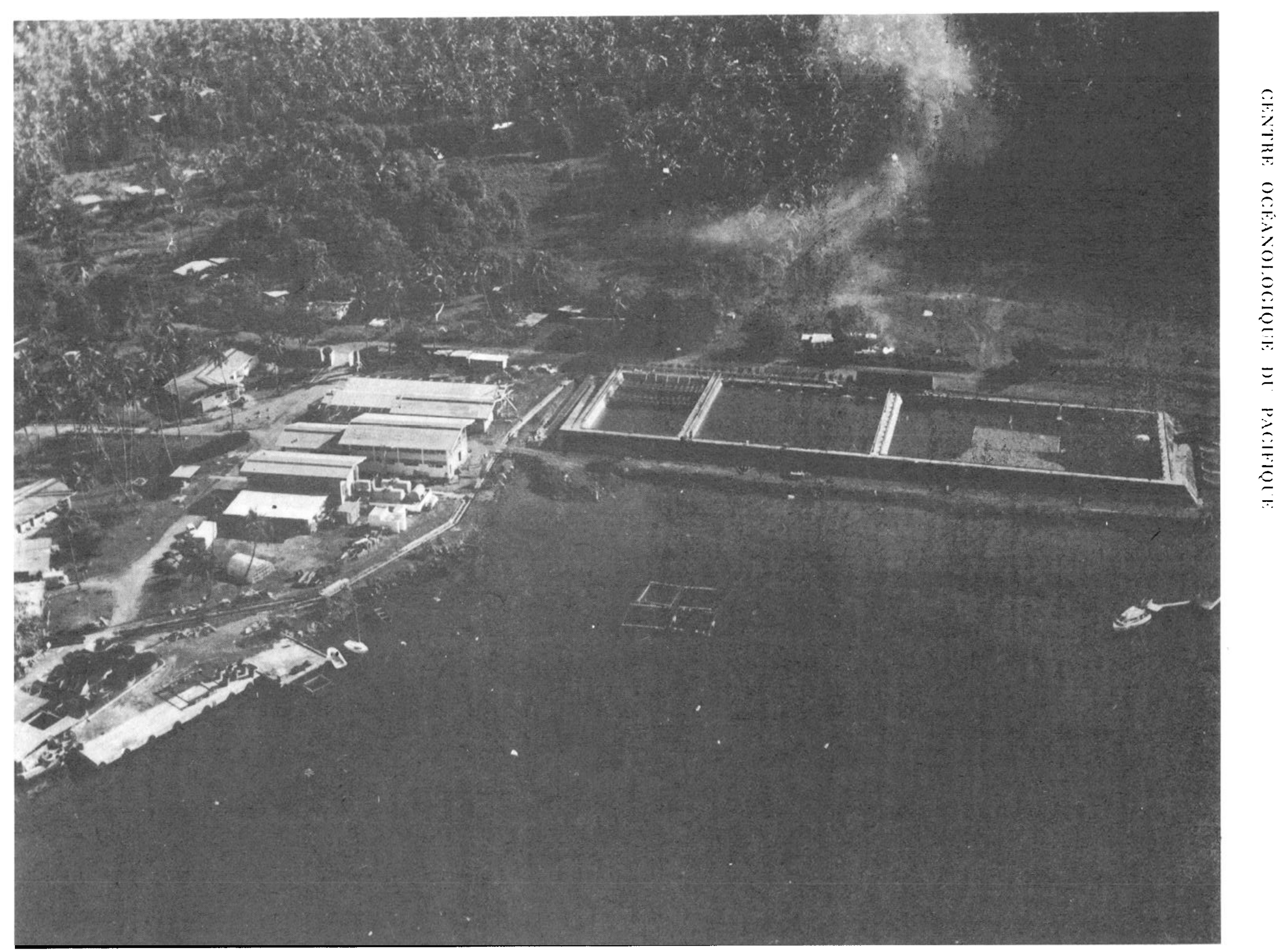

Centre océanologique du Pacifique. Vairao. Tahiti, Polynésie Franģaise. Vue aérienne de Cientre en avril 1975. 


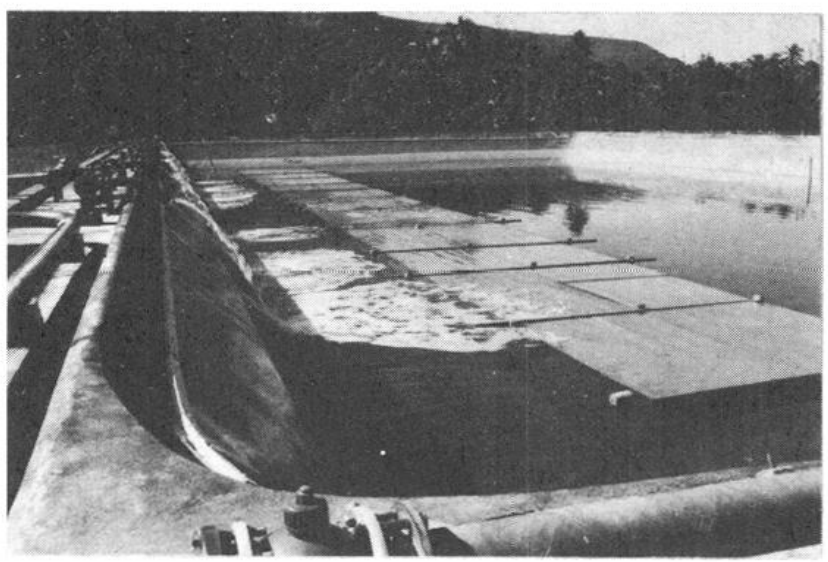

1

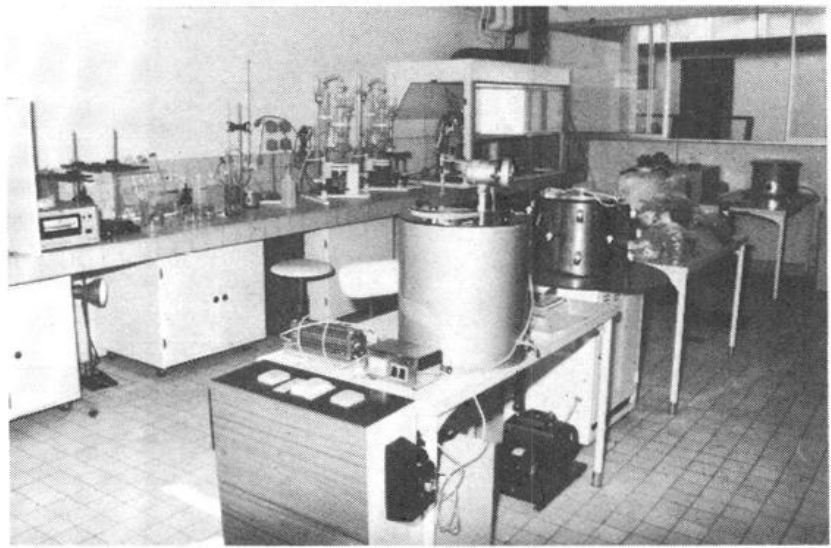

3

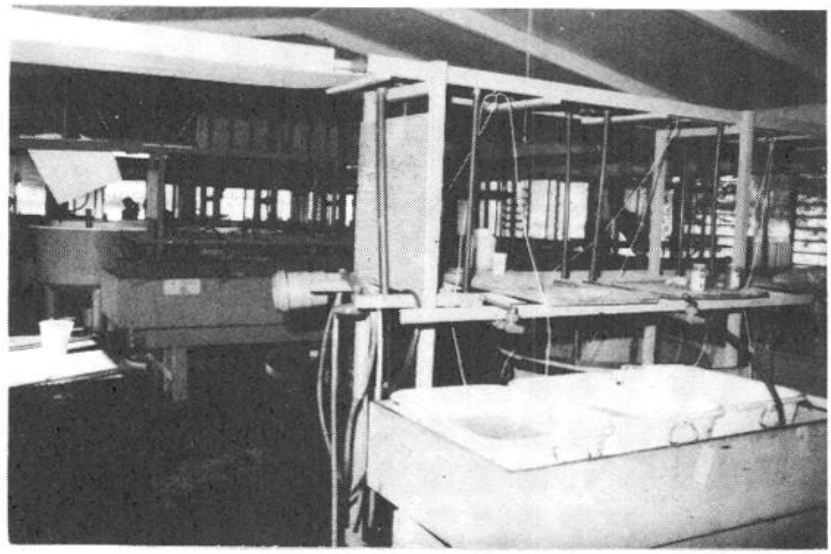

5

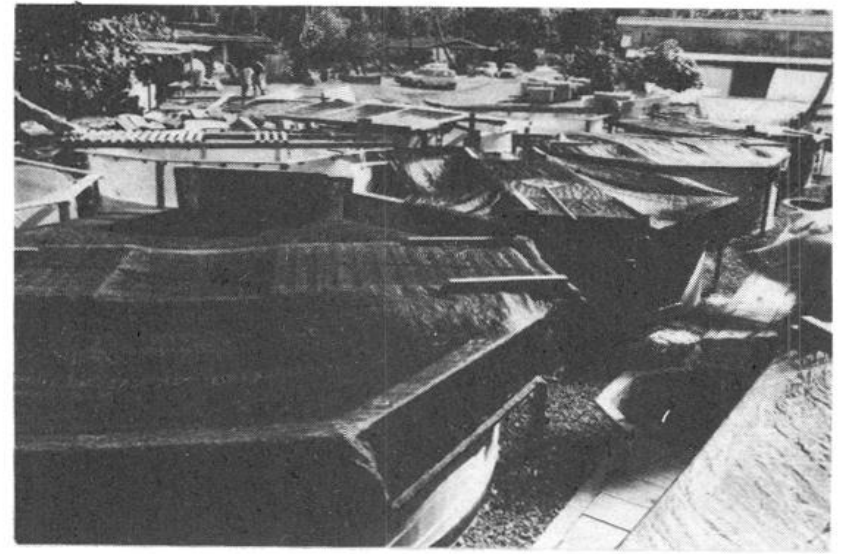

2

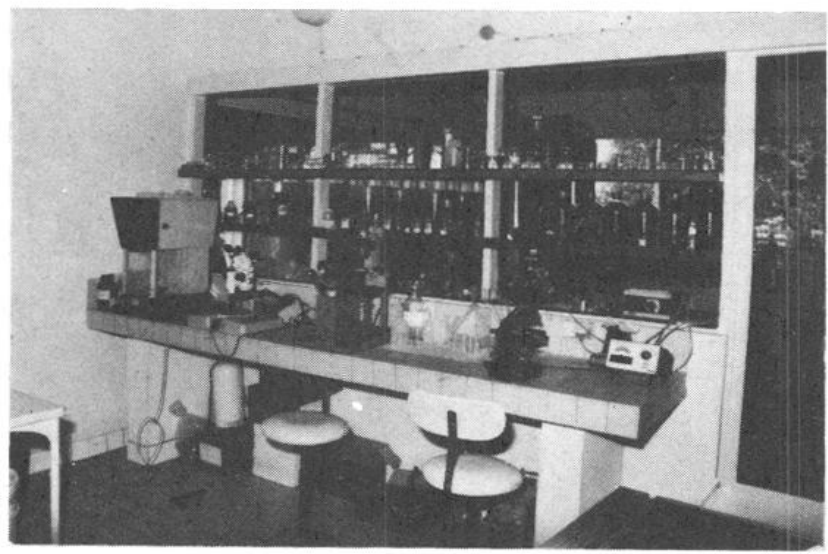

4

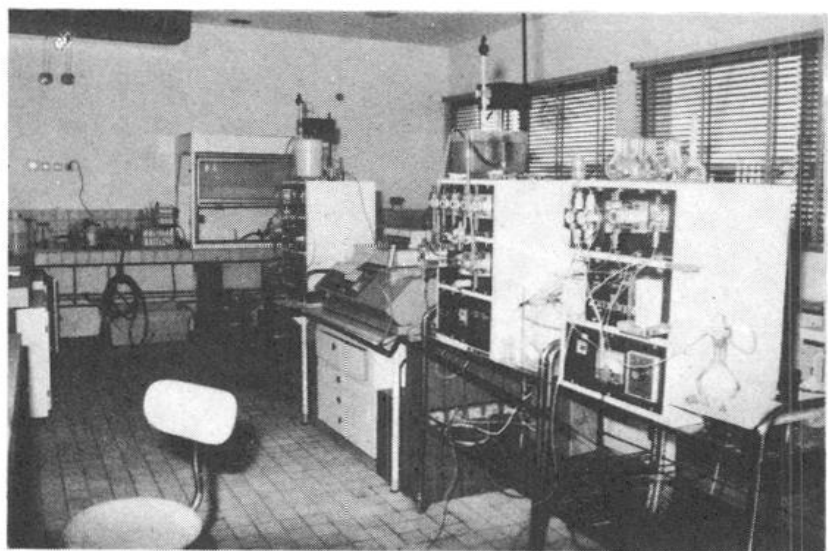

CENTRE OCÉANOLOGIQUE DU PACIFIQUE

1. - Bassin d'élevage. 2. - Bassins à géniteurs. 3. - Laboratoire de nutrition. 4. - Laboratoire d'examen. 5. - Hall d'aquaculture. 6. - Laboratoire de mesures physico-chimiques. 
En aquaculture, le COP s'est fixé de mettre au point à court terme la technique des cultures et élevages, de former le personnel nécessaire, puis de cerner les premiers coûts de production, et susciter des structures ou des initiatives pour aboutir à un début de production de quelques tonnes par an.

Quatre opérations particulières ont été retenues visant à la production de chevrettes, crevettes, poissons et mollusques.

Elles posent toutes des problèmes similaires qui seront résolus au niveau d'opérations plus générales : nutrition (avec comme opérations élémentaires, la production d'algues unicellulaires, de zooplancton et d'aliments composés); mesures; technologie; études de sites d'aquaculture industrielle.

C'est une espèce de chevrettes non représentée dans les rivières de Polynésie qui a été choisie pour être élevée en raison de ses bonnes performances de croissance : il s'agit d'une chevrette d'eau douce originaire de Malaisie, Macrobrachium rosenbergii, qui peut atteindre 200 à 300 grammes. Elle est omnivore, plutôt nocturne, se reproduit en eau saumâtre et dans les estuaires.

Le schéma d'élevage est celui d'un crustacé et comporte 3 phases : élevage larvaire, prégrossissement et grossissement.

Lorsqu'une femelle a été fécondée, l'incubation des œufs retenus sous l'abdomen dure 18 à 19 jours si les températures de l'eau se maintiennent entre 26 et $28^{\circ} \mathrm{C}$. L'élevage larvaire dure de 35 à 45 jours, et donne des " post-larves" que l'on conduit en 30 à 40 jours à un poids d'une centaine de milligrammes. La jeune chevrette est ensuite placée en bassin de grossissement, où elle atteindra 10 à 20 grammes en 6 à 8 mois et sera commercialisée.

L'opération menée en commun avec le Territoire a commencé en février 1973 à partir d'une trentaine de sujets en provenance d'un laboratoire d'Hawaii.

Début 1975, plus de 250000 post-larves avaient été produites, un stock de reproducteurs constitué et réparti à Tahiti en trois endroits différents pour éviter les accidents malencontreux. La production atteignit une tonne et 100000 individus étaient en cours d'élevage.

Si le cycle biologique complet avait été réussi à plusieurs reprises (nous en sommes à la $3^{\mathrm{e}}$ génération), la technique ne peut encore être considérée comme définitivement maîtrisée.

Quatre espèces de crevettes pénaeidées sont élevées au COP, $P$. merguiensis, $M$. ensis, $P$. aztecus et $P$. japonicus. En captivité, le cycle complet a été obtenu pour la première espèce ( $4^{\mathrm{e}}$ génération actuellement) et la reproduction pour les trois autres. L'opération a débuté en octobre 1973 et les premiers sujets d'élevage ont été $P$. merguiensis et $M$. ensis, originaires de Calédonie.

Près de 200000 " juvéniles " ont ainsi vu le jour et les essais de grossissement ont donné au total $400 \mathrm{~kg}$ de sujets commercialisables.

Il reste à acquérir la fiabilité de production, à prévenir les attaques par les champignons et diverses maladies qui peuvent encore décimer les élevages larvaires. Le grossissement en bassin avec ou sans production primaire 
a été entrepris; l'expérimentation continue, et il faudra aborder le grossissement en cages flottantes pour les espèces qui sont susceptibles de se développer dans de telles conditions.

Notons que le COP est le seul centre d'élevage qui fonctionne à partir de crustacés se reproduisant en enceintes contrôlées. Les eaux de la Polynésie ne recélant pas de crevettes, il a fallu obtenir et maitriser maturation et reproduction en captivité.

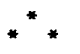

Outre les crustacés, le COP a abordé l'aquaculture des poissons. Le grossissement s'effectue à partir de jeunes sujets, fruit d'une cueillette dans le milieu naturel. Le grossissement s'opère en cage flottante, c'est-à-dire sans être obligé de renouveler l'eau constamment et d'y apporter de l'air pour oxygéner. La production dépend évidemment du volume des captures initiales. Trois cents kilos de carangues ont été produits. Les sujets commercialisés pesaient un peu plus de 300 grammes et convenaient parfaitement aux restaurateurs par leur taille. Ce poids a été obtenu au bout de 6 à 8 mois d'élevage. De grands espoirs sont permis lorsque les aliments nécessaires en quantité suffisante seront assurés.

L'effort en aquaculture de mollusques a été orienté pour obtenir du naissain d'huîtres nacrières (Pinctada margaritifera) et d'huîtres comestibles (Crassostrea gigas).

La fécondation et l'obtention de larves trocophores ont été obtenues pour Pinctada margaritifera; pour Crassostrea glomerata la vie larvaire a été obtenue sans cependant parvenir à la fixation, tandis que pour Crassostrea gigas, la réussite a été complète avec l'obtention de naissain.

Cette opération difficile sera poursuivie pour tenter de pallier le déficit en nacres à greffer et permettre de fournir du naissain de bonne qualité et à croissance rapide aux ostréiculteurs.

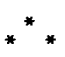

Des essais de pompage d'eau profonde sont programmés. Cette eau riche en sels nutritifs pourra stimuler une production primaire importante à partir de laquelle des protéines végétales en grande quantité seront obtenues. C'est la direction dans laquelle vont s'exercer les efforts dans les années à venir. Du succès de ces travaux dépendra la mise en valeur des lagons tropicaux.

Chacune des opérations qui viennent d'être décrites sont en fait soutenues par des opérations générales, communes à tout élevage.

La plus importante est celle qui a trait à l'alimentation des animaux marins. Une importation totale des ingrédients nécessaires ne saurait être rentable. Les travaux accomplis ont porté sur l'inventaire et l'utilisation des sous-pro- 
duits locaux : drêches et levures de brasserie, tourteaux de coprah, manioc, chair de troca, farine de leucaena sp. Mais ces produits sont déjà utilisés en partie pour les élevages de la terre ferme, et ne sont donc disponibles qu'en quantité limitée. L'orientation donnée aux recherches en cours vise à tirer directement du milieu océanique la majeure partie des ingrédients nécessaires.

Enfin, la définition d'aliments composés s'effectue grâce aux équipements du laboratoire du COP et d'une chaîne de fabrication d'une capacité de $300 \mathrm{~kg}$ par jour.

Autre élément important dans l'alimentation des animaux, la production d'algues unicellulaires est désormais assurée en petits et moyens volumes (100 l), en continu ou en " bloom ".

Les premiers essais sont en cours pour fabriquer de façon artisanale du poisson séché dans les atolls des Tuamotu, dans des séchoirs solaires pour partie avec apport de calorie par les groupes générateur's produisant l'électricité nécessaire au fonctionnement des groupes frigorifiques.

La précision réaliste des programmes en cours, l'allant et la ténacité apportés chaque jour à la solution des problèmes pratiques ou scientifiques et l'esprit d'équipe qui règne au Centre de Vairao font bien augurer du résultat des travaux pour la mise en valeur des ressources maritimes de la Polynésie Française.

Paris, juin 1975.

Jean de CHAZEAUX. 